\title{
THE ROLE OF PALLIATIVE RADIOTHERAPY IN LOCALLY ADVANCED BREAST CANCER REFRACTORY TO NEOADJUVANT CHEMOTHERAPY AND UNRESECTABLE
}

Ana Flávia de Paula Guerra Campedelli', Nilceana Aires Maya Freitas', Thais Franco Simionatto1, Nathalya Ala Yagi', Jean Teixeira de Paiva'1 Carolina Martinelli Bezerril'

${ }^{1}$ Hospital Araújo Jorge - Goiânia (GO), Brazil.

Objectives: Locally advanced breast cancer (LABC) is a major health problem in developing countries, including Brazil. The standard treatment for LABC is neoadjuvant chemotherapy, with or without anti-Her2 therapy, followed by surgery, radiotherapy (RT), and adjuvant systemic treatment if appropriate. However, there are few data in the literature addressing alternatives when neoadjuvant chemotherapy fails to reduce the tumor for surgery. Nearly one third of LABC are resistant to multimodal neoadjuvant treatment remaining unresectable. As an alternative to downsize tumor and allow surgical removal, palliative radiotherapy has been reported as an option. The aim of this study is to verify the role of palliative radiotherapy in the treatment of $\mathrm{LABC}$, once considered unresectable and not responding to neoadjuvant chemotherapy. Methods: It is a retrospective study including 25 patients who had non-metastatic LABC treated with neoadjuvant chemotherapy and who were not eligible for surgical resection; these patients were submitted to salvage radiotherapy between January 2017 and January 2018 at Araujo Jorge Hospital and Cebrom clinic. All patients were followed by photography analysis and by a single observator. Results: During one year, 25 patients were included, with a median age of 55 (30-80) years and the average was 52 years old. The most frequent clinical stages were IIIA and IIIB, corresponding to $18.8 \%$ and $81.2 \%$, respectively, characterized by bleeding and secretion, mostly with pain; mean tumor size was 14 (3-25) $\mathrm{cm}$, and 22 patients $(88.4 \%$ ) had nodal involvement. Neoadjuvant chemotherapeutic regimens were prescribed to $88 \%$ of the patients. Radiation dose initially prescribed was 70 Gy divided into 35 fractions, however the average dose received was 50,2Gy; At the end of radiation therapy, the median tumoral response was 55\% (10-100\%) and the average was $51 \%$. After 104 days post RT the average tumor response was $86 \%$. After 70 days and 120 days post radiotherapy, 2 patients $(8 \%)$ had their tumors downsized and underwent mastectomy, respectively. The overall survival will be still analysed. Conclusion: Neoadjuvant radiotherapy is an effective treatment to downsize breast cancer tumors with low or absent response to chemotherapy, as well as coping with local control. In this study, the radiation treatment was responsible for $86 \%$ of tumor downsize, with great improvement of the bleeding and tumor secretion. 